\title{
Michael Daxner \\ Das neue Hochschulrahmengesetz - (k)eine Hoffnung auf Erneuerung?
}

\begin{abstract}
Der Autor vergleicht die polituschen und hochschulpolitischen Unterschiede zwischen dem ersten HRG von 1976 und den jüngsten Entwuirfen. Kennzeichen des ersten $H R G$ war ein flexibles Zurückweichen des Staates vor den radikalen Konsequenzen der Ende der sechziger Jabre geforderten Reformen. Heute haben sich fast alle Voraussetzungen aus dieser Zett geandert. Die Anpassung der deutschen Hochschulen an internationale Standards ist noch nicht erfolgt. Die partielle Abkoppelung der Hochschule vom lähmenden Einfluß eines finanzschwachen Staates est dringend erforderlich. Der Verfasser zeigt, daß das neue HRG diesen Ansprï̈chen nur zum Teil gerecht werd, eine radikalere Reform geboten ware. Ein entsprechendes Konzept legt seiner Meinung nach der Alternativentwurf vor, der besonders dem Umstand Rechnung trägt, daß bestimmte Bereiche vollständig neu geregelt werden müssen. Die Red.
\end{abstract}

\section{Vorbemerkung}

I. Das Hochschulrahmengeserz (HRG) trat nach langen Beratungen am 30. I. $1976^{1}$ in Kraft, und wurde mehrmals, nachhaltig vor allem $1985^{2}$ und $1987^{3}$ geändert. Weirere kleine Änderungen betrafen marginale Nachbesserungen.

2. Am 20. 10. 1997 wurde durch die Bundesregierung eine total revidierte Fassung cincs HRG in den Bundestag eingebrachr, die auf der Grundlage eines Kompromißpapiers zwischen Bund ${ }^{+}$und Ländern' am 19. 8. 1997 vorgestellt und in der Fassung vom 21.8.1997 ins Gesetzgebungsverfahren gelangt war. ${ }^{6}$ Dicser Text wird hier als Regierungsentwurf bezeichner. Ein Alternativentwurf, der niche als Änderungsgesetz, sondern als eigenständiger Entwurf zum HRG völlig neue Wege beschreiter, wurde am 24. Oktober 1997 von der Parrei Bündnis 9o/Grüne vorgelegr ${ }^{7}$ - in diesem Text als Alternativentw w $f$ bezcichnet. Der Anhörung des Deutschen Bundestages am 8. 12. 1997 lagen noch ältere Resolutionen von SPD und Bündnisgrünen als Marerialien vor, sowie eine allgemein gehaltene Stellungnahme der PDS ${ }^{8}$. Da der Regierungsentwurf die Zustimmung des Bundesrates nicht voraussetzte,

\footnotetext{
1 BGBI. I, S. 185

2 BGBI. I, S. 605 .

3 BGBI. L, S. 1170 .

4 Die Koalition war durch den federfuhrenden Bundesminister Rutegers (CDU) vertreten.

s Die Bundesrats-Mehrheit der SPD-regierten Lander wurde durch Munster Zolhner, Rheinland-Pfalz, vertreten. Insgesamt sind die Landerpositionen nucht enfach entlang der Partelinen zu verorten. Das Abstummungsverhalten der rot-grunen Koalitionen in den Landern (mutrlerweile s) im Bundesrat ist noch ungewiB. Wegen der koordinterenden Rolle von Zollner wird vom Ergebnis mit eingem Recht als cinem Papier der agroßen Koalıtion* gesprochen.

6 BT-Drs. $13 / 8796$

7 BT-Drs, $13 / 8824$

8 BT-Drs. : $3 / 8847$.
} 
kommt der Intervention des Bundesrates ${ }^{2}$ in dieser Hinsicht eine größere Bedeutung zu als nach ihrem eigentlichen Regelungsgehalt, in dem es un die Organisationsparagraphen $\$ \int 60 \mathrm{ff}$. HRG gcht. Interessant ist, daß die studentische Dachorganisation FZS mittlerweile einen eigenen Gesetzentwurf vorgelegt hat, der systematisch dem alten HRG und dem Regierungsentwurf folgt (30.9. 1997). Der Entwurf des sächsischen Wissenschaftsministers aus dem Frühjahr 1997 ist als Druckmittel durchaus wirksam gewesen und etwas innovativer als der Regierungsentwurf, aber nicht ernsthaft in der Diskussion verarbeitet worden.

3. Ich werde zunächst die politische und hochschulpolitische Differenz zrvischen dem ersten HRG und den neuen Entwürfen kurz analysieren. Es geht u. a. darum, Notwendigkeit, Rahmenbedingungen und Folgen der Gesetze bzw. Initiativen zu verdeutlichen. Dann werde ich die heutige, aktuelle Situation genauer belcuchten. Das ist aus zwei Gründen unumgänglich notwendig und auch sinnvoll. Zum einen haben die studentischen Proteste ab Oktober 1997 cine neue Qualität in die hochschulpolitische Diskussion gebracht, zum anderen ist mit der endgültigen Absage an eine Reform der Studienfinanzierung am 18.12. 1997 (Bundeskanzler und Ministerpräsidenten) eine wichtige Weichenstellung gegen eine völlige Neuorientierung der Hochschulpolitikhier ex negativo - erfolgt. Schließlich werde ich die entscheidenden Differenzen zwischen dem Kompromiß des Regierungsentwurfs, den die SPD nachbessern will, und dem Alternativentwurf darstellen und cinige Schlußfolgerungen ziehen. Da ich maßgeblich an dieser Alternative beteiligt bin, werden in diesem dritten Abschnitt die entsprechenden Schlußfolgerungen für die Hochschulpolitik durchaus akzentuiert sein, ebenso in Zusammenhang mit einem Gesetzesentwurf zu einer Alternative zum BAFöG, das die Bündnisgrünen durch eine Fondslösung ablösen wollen.

4. Die rechtswissenschaftliche Kommentierung des HRG ist nicht sehr differenziert ausgefallen. Das muß überraschen angesichts der Tatsache, daß der Prozeß der Verrechtlichung des bundesstaatlichen Hochschulwesens in der Reformperiode 1968I 975 erhebliche öffentliche und politische Aufmerksamkeit gefunden hat. Möglicherweise ist aber gerade darin der Grund zu sehen: Mit der Kodifizierung ist offenbar ein gesellschaftlicher Prozeß zu einem staatlich gesteuerten Abschluß gekommen - genau das wird heute übrigens von den Protest-Protagonisten befürchtet. Teilweise als Autorenkommentar hat sich zunächst derjenige von Dallinger-Bode-Dellian ${ }^{10}$ etablicrt. Der Kommentar von Denninger" hat cine etwas stärker an die sozialliberale Ursprungsgeschichte angelehnte Richtung, wobei einer der Autoren (Henning Schrimpf) auch am neuen Alternativentwurf ${ }^{12}$ maßgeblich mitgewirkt hat. Zu erwähnen sind auch die Kommentare von Großkreutz ${ }^{13}$ und Reich't4. Auch landesgusetzliche Kommentare fallen hochschulpolitisch nicht sehr ins Gewicht. Die Novellierung des Arbcitszeitgesetzes und dic korrespondierenden Änderungen im HRG, vor allem der $\mathbb{S} 57$ a-e, haben noch einmal eine kleine Kommentierungswelle bewirkt (z. B. Nagel's), während die hochschulpolitische Literatur, ohnedies spälich

9 BT-Drs. $13 / 5358$.

10 Dallinger/Bode/Dellaan: Kommentar zum Hochschulrahmengesetz Unter Mitarbeit von Ludwig Giesecke und Klausdeter Tschope, Tubangen: Mohr, 1978.

is Erbard Dennmger: Hochschulrahmengesetz. Kommentar. Bearbeitet von Becker, Denninger, Hammer, Hauck, Kebler, Lennartz, Luthje, Nagel und Schrumpf, Munchen: Beck, 1984.

12 In Zusammenarbeit mit der Bundestagsfrakuon Bundns go/Die Grunen und in deren Auftrag erstellt unter Leitung des Verfassers von Daxner, Gerlof, Hener und Schrompf.

I3 Peter Großkreatz: Kommentar zum Hochschulrahmengesetz. Hamburg: Hettmann, 1978.

14 Andreas Reach: Hochschulrahmengesetz: Kommentar. Bad Honnef: Bock, 1992 (4. Auflage)

is Bembard Nagel: Fristvertrage an Hochschulen und Forschungscinrichtungen: Kommentar. Zugleich Nachtrag zu Denninger. Munchen: Beck, 1986. 
geworden, sich mit dem Gesetz und seinen Auswirkungen nicht mehr grundsätzlich auseinandergesetzt hat.

Weitere Zäsuren in der Diskussion waren 1989 (kurz vor der Einigung, als die Gebührendebatte erstmals hochkochte) und im Zug der Anpassung der Ländergesctze der neuen Bundeslander nach 1990.

In diesem Kommentar werde ich nicht auf die grundsätzliche Frage eingehen, wieweit ein anderer Einigungsvertrag odex eine grundlegende Reformdiskussion andere Weichen hätte stellen sollen oder können - immerhin ist diese Frage in der Hochschulpolitik, auch in der Rektorenkonferenz, ernsthafter gestellt worden, als die rüde Anpassungspolitik mit ihren Ergebnissen heute vermuten läßt. 1996 wurde die Diskussion un die BAFöG-Gesetzgebung ${ }^{16}$ (I 8 . Novelle) und ihren Bezug zum HRG angereichert. Die Trennung der politischen Diskussion vom Prozeß $\beta$ der Verrechrlichung ist in diesem Bercich durchaus bemerkenswert.

I 997 hat sich eine verständliche Verdichtung grauer Literatur und einc Vielzahl von Verbands- und Parteistellungnahmen angehäuft. Die Nachfolgediskussion über die Ländergesetze ist von ausschlaggebender Bcdeutung für die Auswirkungen des neuen HRG, in welcher Fassung auch inumer es beschlossen wird.

\section{Das erste Rabmenrecht}

r. Das erste HRG war ein komplexes Produkt ungleichzeitiger und teilweise kontingenter Entwicklungen. Die Gewichte der einzelnen Begründungen haben sich in der Analyse mit zeitlichem Abstand erheblich verschnben.

$\mathrm{Ab}$ i 968 zogen cinzelnc Länder in ihren Gesetzen Konsequenzen aus der Studentenbewegung, u. a. in den Bereichen Partizipation und Studienreform. Als Endergebnis der bildungsökonomischen Diskussion, dic seit 1958 zunehmend an Gewicht gewann, hat sich die berufsqualifizierende Funktion des Studiums $(\mathbb{2} 2 \mathrm{Abs}$. I und $\$ 7$ HRG) anstelle eines »Gelehrtenideals « durchgesctzt, durchaus gestürzt durch einen breiten gesellschaftlichen Konsens auch mit den Gewerkschaften und der Wirtschaft. Aspekte der Modernisierung und vor allem der Expansion des tertiären Sektors spielten eine große Rolle. Hicrzu stellen wir aber fest, daß nur die Oberfläche einer grundlegenden gesellschaftlichen Veränderung betroffen ist. Darunter liegen wenigstens zwei gewichtigere Motive für das Zustandekommen und die Ausprägung des HRG:

- Das erste Motiv zielt auf die nationalstaatliche Integration wisscnschaftlicher Ausbildung und Hochschulfurschung in ein erstes "Standortkonzcpt" (ohne seine heutige begriffliche Engführung); dazu gchört auch die durchgängigc "Sozialdemokratisierung "(Dahrendorf) des öffentlichen Dienstleistungssektors, die sich im Bestehen auf der staatlichen Einrichting ( $\$ \& \& \mathrm{HRG}$ ) bis heute gehalten hat. In diesem Zusammenhang ist ein Aspekt von überragender Bedeutung, der erst houte wicder in der Diskussion entsprechende Aufmerksamkeit findet. Seit dem Ende der fünfziger Jahre (1957: Sputnik»schock «, I 958: Vollbeschäftigung, 196r: Maucrbau mit entsprechenden Auswirkungen auf die ausgebildetc Arbeitskraftreserve, 1963: OECD-Bericht über die schlechte Position Deutschlands im internationalen Vergleich, 1964: Georg Pichts »Bildungskatastrophe «) wird der tertiäre Sektor selbst zu einem Gegenstand der nationalstaatlichen Reflexion. Bildungsrat, Wissenschaftsrat, eine Reihe von Planungsinstitutionen (HIS u. a.) entstehen, die Kohärenz herstellen 
sollen zugunsten eines einheitlichen nationalen Hochschulsystems, das es vorher de facto nicht gab und nicht geben mußte: Die geringe Beteiligung am Hochschulstudium (lange 7.eit weniger als ı\% ciner Altersstufe) und die geringe Anzahl der Hochschulen ließen sich föderal gut bewältigen. Expansion wurde ab Mitte der Sechziger mit Homogenisicrung gukoppelt. Das Gesetz, das seit Minister Lcussinks Tagen in der Planung war, sollte auch symbolisch der Aufholjagd Westdeutschlands Ausdruck geben und als Integrationsfaktor im Kreis der großen westlichen Industriestaaten wirken. Ich werde darauf zurückkommen, wenn es um die geänderten Koordinaten geht: die abnehmende Bedeutung des Nationalstaates gegenüber supranationalen Verbünden und regionalen Differenzierungen.

- Das zweite Motiv lag in der pazifizierenden Funktion eines modernisierten Rahmengesetzes, das verhindern sollte, daßdie Wissenschaft sich-vcrkürzt-»imDienstedes Volkes « unkontrolliert entfalten konntc, vielmehr sollte sie unter staatlicher Regie ihre hergebrachten Formbestimmungen sorveit wie möglich beibehalten können, d. h. vor allem in der Finanzierung, in der Personalstruktur und im entpolitisierten Charakter ihrer internen Verkehrsformen. Dazu ist es nötig, ein wenig auszuholen.

2. "Wissenschaft in der Demokratie war cin Schlagwort, das vom SDS, dem VDS und vielen Aktiven der sechziger Jahre als Kürzel für einen angestrebten totalen Umbau der Hochschulen verwendet wurde. Es "meint " unmittelbar die Ausweitung der Partizipation aller Hochschulmitglieder, das Ende der Ordinarienherrschaft, dic Mitbeteiligung der Hochschule an der Verwertung von Forschungsergebnissen im Sinne gesellschaftlichen Fortschritts. Damit verbindet es sich mit einem bildungsökonomisch-realen und mit einem teilweise sehr "symbolischen « Aspekt. Nicht nur in der Linken ("Politische Kritik des Ausbildungssektors") war die Frage virulent, was denn hohe Akademisierung zum Fortschritt bzw. Wohlstand einer Gesellschaft im allgemeinen, $z u$ ihrer kritischen und intellektuellen Veränderung im speziellen beitragen könne. Die Produktivität geistiger Arbeit - ein Dauerthema der marxistischen Exegese - traf sich mit der ersten diesbezüglichen bildungsökonomischen Standortbestimmung bürgerlicber Provenienz. Ich möchte die Situation als die kollusive Begegnung zweier unvermittelter Bewcgungen bezeichnen: Die standortorientierte Expansion und das Bedürfnis, über kritische Wissenschaft Teil einer politischen Dynamik zu werden, für dic der alte Wertekatalog weit über die Linke hinaus durch Solidarität, Emanzipation, gesellschaftliche Relevanz ersetzt wurde. Der Bottom-upAspekt der tatsächlichen Reform vereinigte sich mit dem theoretischen top-down der linken Ableiter, im Projektstudium wurde dic gesellschaftliche Relevanz um einen Kern herum gebildet, der im besten Fall so vicle konzentrische Kreise ziehen würde, bis er an den Rand der epochalen Auseinandersetzung (Antiimperialismus, militärisch-wissenschaftlicher Komplex) geraten könnte. Das war gewiß voluntaristisch, aber durchaus vielfach mit guten Einzelbegründungen angestrebt. Durch Kodifizierung meinte die politische Klasse, die modernisierende Potenz gut auffangen zu können, ihre radikale Spitze aber einzufangen - mit Erfolg.

3. Kennzeichen des ersten HRG war im Ergebnis ein flexibles Zurückweichen vor den radikalen Konsequenzen der geforderten Reformen. Alles blieb in der Hochschule und zwischen Hochschule und Staat geregelt. Danit war die schlecht legitimierbare institutionelle Vorherrschaft der Professoren fast unbeschädigt aus der Auseinandersetzung hervorgegangen (unterstützt durch das Bundesverfassungsgericht zu Mitbestimmung und besonderen Privilegien der Professorengruppe ${ }^{17}$ und die

17 Vgl. Denninger, Rz. 1- 52 zu $\$_{43} H R G$ 
enge, individuell bevorzugende Auslegung von Art. s Abs. 3 GG). Man hatte die große Chance verpaßt, die Öffnung ("Bildung als Bürgerrech" ", "Aufstieg durch Bildung ") mit einer öffentlichen Teilhabe am Wissenschaftsprozeß und an der Hochschule zu verbinden. Andererscits kam man dem Trend nach sozialdemokratischer Integration der gesamten Gesellschaft dadurch entgegen, daß man "Lchre und Studium " unsystematisch mit Priorität ins Gesetz setzte (2. Abschnitr des HRG) und die Studienreform in ein bürokratisches Langzeitunternehmen unter staatlicher Homogenisierungsmacht verwandelte. $\mathrm{Da}$ die radikale Linke sich längst von diesem Politikfeld verabschiedet hatte, blich es bci ciner Auseinandersetzung unter den Reformern und gegen die Erviggestrigen. Die Reformer, die z. B. aus der BAK (Bundesassistentenkonferenz) hervorgegangen und überwiegend bei der GEW gelandet waren, bildeten zugleich die kritische Masse, die das HRG vermitteln konnte, und die karrierebewußte Spitze einer breiten Erneuerungsbewegung, die wenigstens Teilerfolge mit dem Gesetz anmelden konnte. Die Hauptanliegen der pragmatischen Reform - gesellschaftsverändernd im Rahmen allgemeiner Bervegungen (mehr Demokratie wagen!) zu wirken - blieben auf der Strecke: echte Gesamthochschulen, einheitlicher Lehrkörper, Verschiebung des wissenschaftlichen Prioritätenspektrums in Richtung auf Relevanz (statt Fachtradition). Die Gruppenuniversität mit ihrem Reformapparat war ein Ergebnis, mit dem man gleichwohl leben konnte. Dennoch setzten sich Bildungswerbung und Hochschulausbau fort, viele der Kritiker wurden so einfach Professoren, wie sie es unter den alten Umständen nie erreicht hätten. $\mathrm{Da}$ paßt man sich gerne an, indem man auch die Illusion nährt, dem Staat eine bestimmte Form von Handeln aufgezwungen zu haben. So gesehen, bietet das alte HRG auch einen nie ausgeschöpften Fundus an unerledigten Reformen (z. B. noch 1996 Elisabeth Altmann, ehemalige hochschulpolitische Sprecherin der Bündnisgrünen).

4. Das Bündnis von Demokratie und 'Technokratie unter den Auspizicn von Expansion und Modernisierung war einerseits insoweit ein "Erfolg", als die mit ihm verbundene institutionelle Absicherung der Hochschulen die Bildungswerbung und Qualifizierungskampagne einigermaßen effizient regelte; vordergründig war mit der Beendigung des deutschen Ordinarienmodells auch ein Schritt zur inneren Demokratisierung gemacht. Hier aber setzt rückblickend die politische Kritik an: Die innere Demokratisierung wurde, soweit es nötig schien, verfestigt (Gremienstruktur der Gruppenuniversitär), aber es wurde keine Brücke zum Demokratiebedarf der Gesellschaft geschlagen. Der "fehlende Republikanismus" des alten HRG rächt sich heute, wenn die Beteiligung von Öffentlichkeit etwa in Form von Kuratorien und Hochschulräten gerade von den "Linken " teilweise vehement abgelehnt wird - mit dem altordinarialen Autonomicargument einer Selbsthestimmung des "Wir wissen am besten, was für uns gut ist «. Der Staat als langer Arm des bildungspolitischen Konsenses ersetzte die Teilhabe der Menschen an »ihrer Wissenschaft«, das Steuerungsmittel war lange Zeit eine additive Ausgabenpolitik. Akzeptanz fand dieses Vorgehen solange, wie "Aufstieg durch Bildung ", d. h. privilegierende Einstellungsbedingungen für Absolventen, massenhaft wirksam erschien. Übersehen wurdc in vielen kritischen Stellungnahmen, daß dic Innenstruktur der Hochschulen, ihre Finanzierung und die Personalstruktur sich nicht nachhaltig veränderten. Unter dem Signet der "Studienreform" (\$\$ 8-9 HRG) wurde eine gigantische, wenig effektive Bürokratie errichtet, deren homogenisierende Wirkung die tatsächlichen Freiräume, die das Gesetz gegenüber dern früheren, willkürlich-lokalen Zustand schuf, wieder einschränkte. Daß die Gesamthochschuloption wieder aus dem Gesetz verschwand, ist charakteristisch für die Veränderungen der konservativen Nachbesserungen nach 1980 . 
s. Für die damalige Ausgangslage der Rezeption und Umsetzung des HRG ist der Status der Kricik interessant, weil sich hieran eine scharfe Differenz zur heutigen Situation profilieren läßt. Ich unterscheide drei Linien dieser Kritik.

- Eine rcaktionärc (nicht konservative) Kritik der Beharrung, die vom Zusammenspiel von gemäßigter Binnendemokratie und quantitativer Expansion Niveauverlust, Finbußen an Forschungslcistung und eine die Wissenschaft einengende Politisierung erwarteten. Die Kritik wurde zwar allenthalben widerlegt - der Erfolg der damaligen Politik hat in Maßen das Gegenteil bewirkt, sie hat aber ihre Spuren bis heute hinterlassen und lebt in neuem Gewand partiell wieder auf (bis hin zu Schwanitz' "Campus «-Roman)' ${ }^{8}$. Wer die Bilanz des notorischen "Bundes Freiheit der Wissenschaft « als Abrechnung mit den 68ern in Dezember 1997 liest ${ }^{19}$, der kann ermessen, wieviel gesellschaftliche Dynamik in der Zumutung liegt, das vorgebliche Humboldt-Modell zu demokratisicren. Exponent war nicht nur der Bund Freiheit der Wissenschaft. Weiter verbrcitct war ein Phänomen, das ich "Gesellschaftsvorbchalt « nenne und das sich im professoralen und juristischen Vorrang der personalen vor der institutionellen Wissenschaftsfreiheit (aus Art. s Abs. 3 des $G G$ ) ausdrückt.

- Eine kapitalismuskritische Ausdünnung der fundamentalen Vorbehalte gegen dic Indienstnahme der Hochschulen durch ein System, das sich durch Notstandsgesetze, Ökonomisierung aller Lebensbereiche, internationale Verflechtung im militär-industriellen Komplex von den Zielen der demokratischen Ausrichtung und Kontrolle der Hochschulen und der Wissenschaft diametral entfernt hat - also dem Paradigma der sechziger Jahre "Hochschule in der Demokratie «" entgegenlief. Typisch für diese Kritik ist die Verbindung enttäuschter Sozialdemokraten mit anderen Anhängern der These vom staatsmonopolistischen Kapitalismus. Repräsentativ für diese Kritik ist etwa der Sammelband von Bracht, Hülsmann, Keiner zum Hochschulrahmengesetz ${ }^{21}$. Die Argumente finden sich bis heute, zunehmend eingebunden in ganz andere - zeitgemäße - globale Kritikfelder, wie Globalisierung, Nachhaltigkeit, Ökologie etc., konserviert, wobei dic Protagonisten, BdWI (Bund demokratischer WissenschaftlerInnen), GEW, studentische Verbände der Linken, oft die neuen Ansätze mit dem alten Vokabular abwerten. Das HRG als Herrschafts- und Formierungsinstrument steht im Mittelpunkt dieser zweiten Kritiklinie.

- Die dritte Linie speist sich aus der Frustration der Hochschulreformer, die über Studienreform ("Projektstudium «), Dienstrecht, Praxisbezug, Öffnung zur Laienkultur ("Wissenschaftsläden«) u. ä. die Verbindung von Modernisierung und Demokratisierung akzeptiert haben, aber im HRG kontraproduktiv verfestigt sehen. Diese "reformistische "Linie - eigentlich ein Konglomerat - solite lange'Lcit einen Rückzug der Hochschulforschung und des Engagements an der lehensweltlichen Ebene der Hochschule erleben, sic spielt heute in veränderter Gestalt wicder cinc große Rolle. Dies um so mehr, als sie die "subjektive Wende“ der sicbziger Jahre nach dem "Theoriehunger « der Sechziger zu integrieren vermochte. (Bei Michael Rutschky heißt dieses Phänomen »Erfahrungshunger «"2.) Die dadurch bewirkte

I8 Detrid Scbwantz: Der Campus. Frankfurt am Man: Eichborn-Verlag, 1995.

19 freihet der wissenschaft, Nr. 4, Dezember 1997 (Themenschwerpunkt).

$20 \mathrm{Vgl}$. den gleichnamigen Leitutel von Nusch, Offe, Prewß, in: Wolfgang Nitsch u. a.: Hochschulc in der Demokratie. Kritische Beitrage zur Erbschaft und Reform der deutschen Universitar, Berlin: Luchterhand, 1965 .

21 Bracht, Hulsmann, Kemer (Hrsg.): Hochschulrahmengesetz, Hochschulpolttk und Klassenausemandersetzungen in der BRD, Koln: prv, 1977.

22 Michael Rutschly. Erfahrungshunger: Ein Essay uber die siebziger Jahre, Köln: Kiepenheuer \& Witsch, 19 So. 
Entpolitisierung etwa der Hochschuldidaktik führt auch zur Marginalisierung dieser Disziplin. Die Kritik dieser dritten Linie hat $\mathrm{m}$. E. auch bewirkt, daß die hochentwickelte und international geschätztc deutsche Hochschulforschung nie so recht in den Status entscheidungsvorbereitender Politikberatung getreten ist, sondern eher legitimatorischen Charakter aufweist.

6. Es ist nicht einfach, die Wirkungsweise eines Bundesrahmengesetzes kausalanalytisch darzustellen, weil ja wesentliche Bestimmungen durch die Ländergesetz.e konkretisiert werden (basierend auf $\$ 72 \mathrm{HRG}$, wobei der Rahmen in der Regel restriktiv und mit Bindungswirkung für die Länder ausgelegt wurde). Dabei spielen im übrigen viele Urreile des Bundesverfassungsgerichts, Bundesverwaltungsgerichts und unterer Instanzen zur Mitbestimmung eine Rolle. Wechselwirkungen mit nicht eindeutiger Richtungsänderung ergaben sich etwa in den Bereichen der Zulassung (Numerus Clausus, Öffnung der Hochschulen für Nicht-Abiturienten) oder in der Studienförderung (mehrere Richrungsänderungen beim BAFöG). Dienst- und arbeitsrechtliche Belange, wie sie vor allem in den \$S 57 a-e HRG nach der ersten Novellierung Ausdruck fanden, haben ihren Ursprung völlig außerhalb der Hochschule und sind eher dem immobilen öffentlichen Dienstrecht sowohl im Beamten- als auch im Angestelltenbereich zuzurechnen.

7. Wie sieht die heutige Situation aus? Es macht wenig Sinn, eine annotierte Chronologie vorzulegen. Wir befinden uns in einem Stadium der Gesellschaftspolitik, in dem fast alle Vorausset7.ungen für eine adäquare Hochschulpolitik und -gesetzgebung sich geändert haben. Der Katalog der wesentlichen Bestimmungen für diese Änderung ist sehr lang, muß aber m. E. akzeptiert werden, un die komplizierte Gemengelage verarbeiten zu können. Ich beschränke mich auf die für die nachfolgende Diskussion relevanten Auschnitte aus diesem Katalog:

- Die Kopplung von wissenschaftlicher Tätigkeit mit gesellschaftlichem Fortschritt, vermittelt über gesellschaftlich sinnvolle Verwertung von Erkenntnis, hat sich unter dem Eindruck der Ambivalenz von Wissenschaft aufgelöst.

- Die Hochschule droht ihre Zentralität in Studium und Forschung zu verlieren, d. h. die institutionelle Einheit aller wissenschaftlichen Tätigkeiten droht effektiveren Teilinstitutionen zu weichen.

- Die materielle Privilegierung durch den Hochscbulabschluß nimmt drastisch ab, die symbolische hält auf absehbare Zeit noch abgeschwächt vor.

- Das deutsche Hochschulsystem als nationalstaatliches wird zunehmend durch die Systemkonkurrenz, vor allem die angelsächsische, in den neu entstandenen zentralund osteuropäischen Staaten unter Druck gesetzt. Die Auswirkungen supranationaler Entwicklungen (7. B. Maastricht- und Amsterdam-Verträge, Ol:CD, UNESCO-Konvention über Anerkennung von Studienleistungen, Forschungsglobalisierung) und regionaler Verselbständigungen (mehr als 20 derartiger Verbünde in Europa) sind unübersehbar (auch darin liegt die abnehmende Attraktivität des deutschen Systems für ausländische Partner und Studierende).

- Das System ist - auf hohem Niveau - chronisch unterfinanziert, seit 2s Jahren stagnieren die Nettoaufwendungen für Hochschulen (außer Medizin).

- Die Personalstruktur ist leistungsfeindlich und widerspricht den europäisch weitgehend durchgesetzten Mindeststandards effektiver Organisation.

- Das Studium ist schlecht strukturiert und bieter zu wenig differenzierte Abschlüssc, sowohl was erwartete Tätigkeitsmerkmale als auch biographische Multipolaritäten betrifte.

Wären diese Phänomene für sich genommen noch Anlässe partieller und unter 
Schwierigkeiten möglicher Reparaturen, gibt es weitere, die eine grundlegende Reform nahelegen. Dic abnchmende Kompetenz der staatlichen Wissenschaftsverwaltung und -steuerung legt eine "Entstaatlichung nahe, die allerdings nicht mit der neoliberalen reinen Marktförmigkeit öffentlicher Dienstleistungen ineinsgesetzt werden darf. Gleichzeitig hat die Lebenslüge von der Autonomie der deutschen Universität ihre Endphase erreicht: Die Forderung nach sechter", d. h. praktischer Autonomie und gleichzeitiger Verantwortlichkeit für das institutionelle Handeln in den Bereichen Finanzen, Organisation, Abschlußdifferenzierung und Personalentwicklung ist nicht mehr rückgängig zu machen. Nebeneffekte der deutschen Vereinigung, das Zusammenbrechen der Studienfinanzierung und dic danit verbundenc sozialc Frage verschärfen den Problemlösungsdruck. Unvermitrelt sind wir in einc Situation geraten, in der von einem neuen Rahmengesetz mehr als nur regulative Impulse oder ein ordnungspolitisches Signal crwartet werden: Das Gesctz sollte einen Anstoß zu einem Element auch gesellschaftlicher Frneuerung von und durch Wissenschaft geben. Das ist eine politisierte Hoffnung.

\section{Bedingungen für ein neues Rabmenrecht}

1. Der Katalog der Rahmenbedingungen für ein neues Gesetz ist bereits umfangreich genug: Dennoch erklärt er nicht, warum - fast durch eine List der Vernunft - die Diskussion um ein neues HRG plötzlich an Dynamik und Intensität gewonnen hat. Es geht dabei um eine Mehrzahl von relativ unabhängigen Entwicklungen unterschiedlicher Intensität, die koinzidieren.

Das alte HRG war weder in der Lage, die reale hochschulpolitische Entwicklung zu antizipieren und durch Anpassung zu gestalten, noch regelt es seigentlich «, was es rahınenrechtlich hätce regeln sollen. Das mußte sich spätestens zu dem Zeitpunkt rächen, als die wirklichen Bedingungen der Hochschule weder administrativ noch durch externe Steuerungsmechanismen, vor allem Geld und restriktive VerwaltungsmaRnahmen, in den Griff zu bekommen waren.

Ich stelle zunächst einige Thesen auf, warum gerade jetzt die HRG-Reform aus ihrer Latenz getreten ist:

a. Der Nachholbedarf gegenüber dem westlichen Ausland und gegenüber der Erwartungshaltung der neuen östlichen Demokratien gegenüber dem erfolgreichen Modell Deutschland ist zu groß geworden, um mit dem "bewährten Erfolgsmodell « ohne Phrase weiterhin operieren zu können.

In den meisten westlichen Ländern ist die Anpassung an moderne Strukturen - noch ohne Wertung bezüglich der tatsächlichen »Modernität« - seit etwa is Jahren im vollen Gang oder abgeschlossen. Die Stichworte dazu sind: Accountability, Autonomy, Accrcditation, Lump sum budget, Modularization. Ich gebrauche nicht ohne Ironie die englischen Fachausdrücke, weil sic teilwcise schlecht ins Duutsche zu übersetzen sind und nicht zufällig aus der Idiomatik pragmatischer Ökonomie entlehnt scheinen. Sowohl dic sogenannte "managerialistische" Schule als auch die Auffassung von der Hoihschule als Unternehmen setzten sich in Ländern wie Großbritannien, den Nicderlanden, Neuseeland, Australien, Finnland und den skandinavischen I.ändern damit durch, die Institution wie ein Unternehmen, aber nicht als Geschäftsbetrieb zu führen. Damit war klar, daß die staatliche Adniniscration weitgehend ausgedient hatte und daß die Hochschule sich ihren "Stakeholders" verpflichtet fühlen müßte, Effizienz und Effektivität nachzuweisen. (Die Ironie des 
schwer zu übertragenden englischen Begriffs ist, daß seine Nähe zu den "Shareholders" gewollt ist, aber auch dic Distanz markiert: Das öffentliche Eigentum an Hochschulen ist hier mit dem Interesse gepaart.) Aber wie schafft man den Nachweis, reguliert durch das Recht?

Wir werden im Regierungsentwurf und im Alternativentwurf sehr unterschiedliche Beispiele für die Lösungsstratcgien finden, aber beiden neuen Entwürfen ist gemeinsam, daß sic die Grundzüge dieser Entwicklung akzeptieren, wenn auch in recht unterschiedlicher Schwerpunktsetzung und Gewichtung. Keiner der beiden Entwürfe verzichtet auf die Setzung, daß es des Rechts bedarf, um den Bereich zu regeln. Ich verweise auf die Bcreiche Bewertung, Leistungsfinanzierung, Akkreditierung und Studium bzw. Studienreform in beiden Vorlagen. Ob sie rechtlich geregelt werden müssen, ist nicht dogmatisch zu bcjahen. Wenn radikale Alternativen beschritten würden, müßten sie in einer neuartigen Verknüpfung von Autonomie und Selbstverwaltung liegen, die allenfalls die Marktregulierung und den Zugang sowie die Abschlußlizensicrung rechtlichen Kategorien unterwürfe.

b. Die Entstaarlichung hat auch noch einen zweiten Aspekt, den ich den zivilgesellschaftlichen nenne. Das HRG subsumiert den gesellschaftlichen Zweck und den Auftrag der Hochschulc unter die hoheitliche Superstruktur staatszweckorientierten Handelns. Das ist nicht länger aufrechtzuerhalten, die Tendenz geht zu einem stärkeren Absetzen der Gesellschaft vom staatlichen Regulierungshandeln.

Es ist auffällig, wic unterentwickelt die Aufgabensetzung und der Auftrag der Hochschulen im HRG (vor allem $\$ 2$ ) geregelt waren, wenn man die gesellschaftlichen Diskussionen der damaligen Zeit beachtet. Auch der Regierungsentwurf hat hier noch nicht viel zugelegt, trifft sich aber in $\$ 58$ mit den Bestimmungen von $\$ 13 \mathrm{Abs}$. I Alternativentwurf, wonach Hochschulen nicht mehr zwingend "staatliche Einrichtungen « sein müssen, um Körperschaften öffentlichen Rechts zu sein oder eine andere Rechtsform anzunehmen, dic ihrem Auftrag entspricht (gerade die Aufgaben- und Auftragsetzung ist nur in $\$ \$ \mathbb{S}^{-6}$ Alternativentwurf relativ ausführlich erfolgt, urn deutlich zu machen, welchen Rahmen man einer zentralen Institution des bürgerlichen Staates geben möchte).

c. Die Aufgabenkritik ist ein Beispiel für den Funktionswandel des Gesetzes. Die A bschnittc über Studium und Lehre sind ein anderes. In schöner Offenheit bictet die Begründung des Regierungsentwurfs ein ganzes Bündel unbewältigtcr Probleme. Warum müssen gerade Studium und Lehre bundesrechtlich so weitgchend reguliert werden, wo doch hier das Versagen der Bund-Länder-Koordination besonders evident ist? Als erstes wird bei der Begründung die Mobilität der Studierenden innerhalb des Bundesgebiets genannt, als zweites die Rechtseinheit. Allc Reformgesetze sind auf Mobilität innerhalb konkurrierender Systeme mit hoher Profilqualität der einzelnen Studiengänge an eincr bestimmten Hochschule ausgerichtet, Konformität innerhalb eines Landes ist Sache des dortigen Gesetzgebers, und der Zwang zu übcrnationalen Orientierungen triftt gerade nicht den Gesetzgeber, sondern meistens die einzelne Hochschule. Die beiden Begründungen "Rechtseinheit « und »Mobilität« sind, neben cinigen Sätzen der Rechtsprechung, die Hauptargumente für das Belassen der Grundstruktur der »deutschen« Universität im Bereich der Personalstruktur, des Dienstrechts und der Hochschulzulassung. Nur im Organisationsbereich (\$\$ s8-66 Regierungsentwurf) meint die Bundesregierung, alle Optionen für die Länder freigeben zu können, und summiert darunter - systematisch bedenklich-gleich auch die Rechtsstellung ( $\$ 58$ Regierungsentwurf).

2. Die heutige Gesetzgebung muß, anders als vor 1975 , von einem voll entwickelten, noch immer sehr leistungsfähigen, aber strukturell vcralteten Hochschulsystem aus- 
gehen, das an eine Reihe von Veränderungen angepaßt werden muß und das eine Reihe weiterer Reformen selbst anstoßen sollte.

a. Anpassungsdruck besteht in der Öffnung zum internationalen Standard sowohl für Studierende als auch für die Laufbahnen der wissenschaftlich 'Tätigen. Hochschulen waren zwar immer - wenigstens ideell - universal ausgerichtet, aber erst durch dic Auflösung nationalstaatlicher Souveränität in supranationale Einheiten und regionale Substrukturen spielt dieser Aspekt der Globalisierung eine unabweisbare Rolle.

b. Die Funktion als Bildungswerbungsgesetz ist obsolet, die Berufsorientierung geknüpft an die Verbindung von Abschlüssen mit Laufbahnen, vor allem des öffentlichen Dienstes, gegen jede Effektivität und Arbeitsmarktresonanz gerichtet; dafür muß ein neues Gesetz die marktwirtschaftlichen Rahmenbedingungen einer viel stärker auf Wettbewerb im Markt gerichteten individuellen Nutzung des Hochschulstudiums, nicht nur der Abschlüsse, sozial regeln.

c. Die studentische Normalbiographie der Nachkriegszeit - männlich, berufsorientiert, aufstiegs - und statusbewußt - ist längst einer multipolaren Biographie gewichen, bei der nicht nur die Seite der Frauen das Nachtrageprofil total verändert hat. Für diese Differenzierung bietet das deutsche System bisher keine Entsprechung auf der Angebotsseite. Zwei formal ultrahomogene Hochschultypen (Universitäten und Fachhochschulen) beherrschen den Abschlußmarkt mit gerade zwei universitären Abschlüssen (Diplom, Magister) und einem Fachhochschuldiplom. Auch die Staatsexamina-Karrieren widersprechen den neuen Marktgegebenheiten. Die differenzierte Nachfrage und der Wunsch, nicht als Drop-out zu landen, sind ja marktförmige Antworten auf ein staatsverwaltetes Monopol.

d. Autonomie soll in Zukuntt die des Unternehmens (enterprisc) und nicht des Geschäfrsbetriebs (business) sein. Darin besteht, bis auf wenige Residuen hoheitlich-besitzstandswahrenden Staatsglaubens, Einigkeit. Aber das bedeutet auch, neben wirtschafulicher Betriebsführung, volle Personalhoheit, Haushaltshoheit und Operarionsfreiheit, also eine Übernahme von Risiko und Hattung für Fehlentscheidungen durch die Hochschule zu fordern. Daran knüpfen sich eine Reihe von sehr pragmatischen Forderungen an Organisation (transparent, wirtschaftlich, flexibel) und Leitungsstruktur (professionell, effektiv, mit starker Legitimation). Aber es ergeben sich noch vicl wichtigere Folgerungen für dic Willensbildung und die Entschcidungsstrukturen in der Hochschule und zunehmend gemeinsam mit der Öffentlichkeit (Kuratorien, Akkrediticrung). Jedenfalls sollen Fntscheidungen weder den Professoren noch der Verwaltung allein überlassen bleiben.

e. $\mathrm{Daß}$ das Beantenrecht und der BAT für wissenschaftliche Arbeit nicht regulierungsfähig sind, ist trivial. Wie weit kann aber ein neues Dienstrecht auf cine erneuerte Personalstruktur aufbauen? Der Regierungsentwurf beantwortet die Frage gar nicht, der Alternativentwurf geht bis an die Grenzen der Verfassungsänderung. Entscheidend ist aber, ob wissenschaftliche Tätigkeit überwiegend die Verbindung von bcruflicher Wahl und Tätigkeit innerhalb der Staatszwecke darscellt oder ob Studium und Forschung in eine neue Beziehung zum öffentlichen Eigentümer der Hochschulen treten müssen, um Zukunft zu gestalten (Politik) und zu gestatten (nächsre Gcneration).

f. Gerade an dieser letzten Option - für das öffentliche Eigentum an Hochschulen kann man die derzeitige politische Diskussion gut festmachen. Eine Gesellschaft darf ihre Hochschulen nicht einfach beiseitelegen, in einer Art Überdruß, gespeist aus Geldmangel, Inkompetenz der Verantwortlichen und einer nicht in die Zukunft ausgreifenden Besitzstandort-Logik. Genau das aber geschicht, und die protesticrenden Studenten haben cines genau gemerkt: Sic sollen erneut verladen werden. Man rationalisiert und spart auf ihre Kosten, ohne daß das System in irgendeinem Punkt 
effektiver, billiger oder leistungsfähiger würde. Die bloß organisatorische Effizienz führt geradezu zu einem Betonieren der bestehenden Machtstrukturen, und damit sind nicht nur dic Professoren gemeint.

Wo die Sudenten »lebensweltlich « argumentieren, vor allem im Ausgangsland des Streiks, in Hessen, stehen zunächst die realen Bedingungen - volle Veranstaltungen, wenig Lchrende, zu wenig Bücher - im Vordergrund. Das "Recht auf Studium « wird eingefordert. Wo die Studenten aber "gesellschaf tspolitisch" argumentieren, steht das HRG im Zentrum der Proteste (Nordrhein-Westfalen), oder es werden Forderungen nach mehr und substantiierter Mirbcstimmung, politischem Mandat etc. sofort zur Überbauung der Proteste benutzt. Das ist insofern für den konkreten Anlaß dieses Kommentars von Bedeutung, als die "lehensweltorientierte u und die dritte, die "Zukunfrs"-Fraktion, von vurnherein von geserzlichen und staatlichen Regelungen weniger halten, sondern sich »ilure Hochschule erobern wollen. Es sind auch Studenten, die »ihre Professuren « behalten wollen, wenn sich anderswo schon die Kritik an Macht und Geld dicser Gruppe zu einer Frontstellung entwickelt. Dagegen stellen die Partizipationisten der zwciten Gruppe starke etatistische liorderungen an einen demokratisch reformierten Staat und seine Exekutive, während sie höchst verkürzt die Öffentlichkeit - also allc, die bei ciner Körperschaft etwa in Kuratorien, Hochschulräten usw. mitbestimmen dürfen - unter »Wirtschaftsverdacht" stellen. Konsequent ist die Gruppe gegen jede Legitimation der Hochschulen vor der Öffentliclakeit durch Leistungskriterien und indikatorgcsteuerte Finanzierung cingestellt.

Die "7 ukunftstraktion « ist insoweit besonders wichtig, als sie den jetzigen Protesten das Gewicht der Anklage gibt: "Ihr, die Erwachsenen, die 68er, die politische Klasse, habt uns die Zukunft verbaut. Jetzt wollt ihr uns auch noch unter ein Leistungsdiktat stellen, das in die Sackgasse führen muß."

Die drei Gruppen sind erstaunlich genau abzugrenzen, wenn man sich auf die Abstimmungsergebnisse der Streikresolutionen und die Aussagen der Wortführer konzentricrt. An der Basis sieht das heterogen aus, aber - das ist für die Funktionärskader alten Zuschnitts neu - es gibt eine derartige Basis. Mit fortschreitendem Protest haben zwar die alten Funktionäre noch einmal die Syntax der Resolutionsrhetorik in den Griff bekommen, aber ihre Legitimation bei den Betroffenen ist gesunken. Leicht crklärlich ist, daß gerade die SPD-Jusos den etatistischen Strang ihrer Partei verstärkten (z. B. bei den Ministern Zöllner und Frau Brunn, dagegen votiert nur der Ncurcktor Pcter Glotz); weniger verständlich ist, daß eine Reihe grüner Hochschulgruppen geradezu realsozialistische Kritik am Nlternativentwurf übt und die etatistische Variante fast deckungsgleich mit dem BdWI, einem der realsozialistischen Üburlcbenden der alten BRD, gegcn die Parteibeschlüsse und Gesetzesvorlagen (auch zur Studienfinanzierung) wenden. Eine genaue Analyse dieser studentischen Posicionen steht noch aus, sie ist nur in bezug auf das HRG hier von Bedeutung. Wir können einen stark anti-autonomistischen Zug erkennen, und zwar sowohl bei den Etatisten als auch bei denjenigen Lebensweltlern, denen die Professorenvormacht an sich ein Grund zum anti-autoritären Aufbegehren ist: Für diese Gruppen bedeutct Autonomie Ordinarienautonomie und Forschungspriorität gegenüber der Lehre bzw. dem Engagement für die Studierenden. Hingegen wollen die zukunftsorientierten und die pragmatischen Lebensweltler sehr wohl die Autonomie der Institucion und einc Öffnung gegenüber der Öffentlichkcit, weil sie sich von dort -z. B. durch Hochschulräte oder die Berliner Kuratorienerfahrung - Unterstützung und Kontrolle gegenüber dem Closed-shop-Verhalten der Hochschule erhoffen.

Für vicle studentische Vertretungen ist vor allem das HRG zum Symbol des Kampfes, besser des Widerstands geworden, weil sich an ihm und an der gescheiterten BAFöG- 
Regelung die Unehrlichkeit der Politiker zeigt, die zwar die studentischen Proteste "an sich «loben, um sich zu entlasten, aber de facto eine andere Sprache sprechen: Studiengcbühren, Leistungsrechnung fast ausschließlich auf dem Rücken studentischer Leistungen, Auswahlrecht der Hochschulen ohne Gegenleistung für die nachfragenden Studenten - das ist die eigentlich konservative Botschaft der politischen Klasse und des Regierungsentwurts.

Vor diesem Hintergrund erfolgt nun eine Gegenüberstellung von Regierungsentwurf und Alternativentwurf, wobci einige Schwerpunkte vor die Klammer gezogen werden.

\section{Zwei Gesetzentwürfe, beinabe zwici Welten}

x. Im Aufbau hält sich der Regierungisentwurf an die Gliederung des ersten HRG. Weil aber nun im Lauf der Zeit schon vor dem jer\%igen Entwurf vielc Änderungen und Ergänzungen vorgenommen wurden, ist das l,rgebnis denkbar unübersichtlich, zumal einige Probleme einfach durch "Wcglall eincr Scheinlösung nahegebracht wurden: Gestrichen werden viele Vorschriften ${ }^{2 y}$. Diese Unübersichtlichkeit hat in der Kürze der Zeit bis zur Anhörung am 8. 1 2. 1997 dazu geführt, daß viele Details - fast alle verbliebenen Paragraphen wurden wenigstens redaktionell geändert - crst nach und nach identifiziert und analysiert werden.

Demgegenüber legt der A.lernativenrwurf eine grundlegend neue Gliederung vor, die vor allem dem Umstand Rechnung trägt, daß bestimmte Regelungsbereiche neu geordnet werden müssen (Akkrediticrung in den $\$ \mathbb{\$} 6-8$, die Hochschulverfassung in den $\mathbb{S}$ i3-rs etc.), während andere besonders herausgehoben werden, z. B. die Frauenförderung als eigener Abschnitt in einem $\$ 27$. Der Alternativentwurf umfaßt insgesamt nur 49 Paragraphen, obwohl eine Reihe von zusätylichen Regelungen gegenüber dem bisherigen Rahmenrecht aufgenommen wurde, z. B. zur Akkreditierung, zu den Hochschulkuratorien und zu einer neuen Personalstruktur.

2. Im Verhältnis zwischen Bund, Ländern und Hochschulen ișt der Regicrungsentwurf auf eine stärkere Bundeskompetenz ausgerichtet, beginnend bei der Leugnung der Zustimmungspflich des Bundesrates (m. E. eine taktische Verhandlungsposition, die bei den Bundesrats-Beratungen gegen etwas anderes eingehandelt wird, weil an der Zustimmungspflicht kcin Zweifel besteht). Der Alternativentwurf hingegen setzt auf starke Ländergesetzgebung mit dem Druck, bestimmte bundesrechtliche Regelungen direkt an die Horchschulen weitcrzugeben. Ein Beispiel: Während die SPDMehrheir des Bundesrates in Bezug auf die Organisation eine "Experimentierklausel « fordert, legr der Alternativentwurt uinige wenige Essentials bundesrechtlich fest und verhindert damit, daß dic Länder bestimmte gewollte Fortschritte rückgängig machen oder relativieren (z. B. Stimngewicht der Studierenden bei der Leitungswahl).

3. Relariv große Übereinstimmung \% wischen den beiden Geserzentwürfen besteht in folgenden Bereichen:

a. Zur Rechtsstellung ${ }^{24}$, wobei der Alternariventwurf grundsärzlich davon ausgeht, daß die Hochschulen keine staarlichen Finr: hrungen mehr sein müssen, während der Regierungsentwurf bloß dic Uption auf Freiheir vom ' $\angle$ wang zur Anstaltsverfassung

23 Die $\int 921,23,28,33$ a, 38-40, $51,57,60-69,74,75,75$ a, 82 fallen weg. Die hochst relevanten Dienstrechtsbestimmungen der $\$ \subseteq S 7$ a $-f$ bleaben erhalten.

24 Regierungsentwurf $\$ 58$, Alternativentwurf $\$ 13$. 
bejaht; die körperschaftliche Verfassung ohne Anstaltszwang ist aber ein besonders umstrittenes Anliegen der Reformer in beiden Lagern.

b. Zur staatlichen Finanzierungspflicht nach Leistungskriterien ${ }^{25}$ : Die Unterschiede liegen hier in den sehr unterschiedlichen Kriterien und darin, daß die Besvertung (Evaluation) beim Alternativentwurf ausdrücklich eine andere Funktion als die Indikatorenfeststellung hat (Alternativentwurf $\$ 18$ ). Auch das Akkreditierungsverfahren (Alternativentwurf $\$ \$ 6-8$ ) hat bewrertende Elemente, hier geht es allerdings nicht um die Finanzierungspflicht, sondern u. a. um die Finanzkraft des Hochschulträgers.

c. Zur Einführung international kompatibler Masters- und Bachelor-Studiengänge ${ }^{26}$ : Allerdings orientiert sich der Regierungsentwurf nach wie vor an Regelstudienzeiten, die beim Alrernativentwurf keine Rolle mehr spielen, wie überhaupr der Alternativentwurf das "berufsqualifizierende "Abschlußziel des Regierungsentwurfs in Frage stellt und tätigkeitsorientiert verfährt.

d. Aus unterschicdlichen Gründen verzichten Regierungsentwurf und Alternativentwurf auf eine Definition der Hochschularten. Die Regierung sieht sich dem Konkurrenzdruck der Fachhochschulen gegenüber den Universitäten ausgesetzt, die teilweise bereits jetzt erfolgreich an diesen vorbeiziehen und durch eine notwendigerweise enge Regelung an dieser Strategie gehindert würden. Die Grünen wollten Übergänge zwischen den Hochschularten wohl erwägen, wurden aber von ihrer universitären Klientel gebremst, weil sich bei fließsenden Übergängen auch einc Differenzierung der rvissenschaftlichen Hochschulen anbietet. Das geht vielen noch zu weit. So bleibr es beim informellen Ranking.

4. Die wesentlichen Unterschiede der Entwürfe liegen, neben der anderen Gesetzesstruktur, in folgenden Bereichen:

a. in der Konstitution der Hochschulen und Studiengänge durch Akkreditierung (statt wie bisher durch cxckutive Genehmigung seitens des Staates) ${ }^{27}$;

b. in der verpflichtenden Einführung von Kuratorien zur Sicherstellung der Mitwirkung der Öffentlichkeit ${ }^{28}$;

c. in einer völlig neuen mitgliedschaftsrechtlichen und dienstrechrlichen Regelung für die Personalstruktur ${ }^{29}$ Diese löst $\mathrm{m}$. E. einige "gordische Knoten " und beruht auf folgenden Prinzipien: Nach der Qualifikation zur wissenschaftlichen Tätigkeit (befristet, Zicl Promotion, Alternativentwurf $\$ 23)$ erfolgt $(\mathrm{k})$ eine Aufnahme in cinen cinheitlichen Lehrkörper ${ }^{30}$, teilweisc in Anlehnung an die Vorstellungen der damaligen Bundesassistentenkonferenz (BAK) im Kreuznacher Konzept. Die Übernahme in den Lehrkörper erfolgt zunächst auf Probe. Eine dauerhafte Übernahme danach eröffnet eine mit zunehmendem Dienstalter immer weniger attraktive Tätigkeit, cs sei denn, sie würde durch Leistungszulagen finanziell aufgebessert oder es kommt zu einer Berufung als Professorin oder Professor (Alternativentwurf \$22). Eine Erstberufung, etwa nach den heutigen Kriterien, erfolgt ebenfalls auf Zeit, danach kann nur mehr im Regelfall auf Dauerstcllen berufen werden. Nicht erfolgrciche Probezeiten - dic Ausinahme - führen zu einer Weiterbeschäftigung auf dem vorigen Niveau. Differenzierende Dienstgrade - heute cin Chaos von höchst laufbahnschädlicher Absrufung - entfallen. Ein solches Modell - hier wurde die Grundform verkürzt dargestellt - kann nur funkrionieren, wenn Beamtenrecht und BAT gleicher-

25 Regierungsentivurf $\$ \$ S$ und 6 . Alternativentwurf $\$ \$ 11$ und 12 .

26 Regierungsentwurf $\$ 19$, Alcemativentwurf $\$ 37$.

27 Alternativentwurf $\$ \$ 6-8$.

28 Alternativentwurf $\$ \$ 9$ und 10 .

29 Alternativentwurf $\$ \$ 19-24$.

30 Alternativentwurf $\int 2 \mathrm{I}$. 
maßen außer Kraft gesetzc werden und ein neues, auf wissenschaftliche Tätigkeit in Hochschulen ausgerichtetes Dienstrecht cingeführt wird, dessen Ausgestaltung den Tarifpartnern mit den Hochschulen als "Arbeitgebern« überlassen wird (Alternativentwurf $\mathbb{2} 24, \mathbb{4} 8$ Abs. 2). Ein Teil dieser Regelungen muß landesspezifisch differenziert werden, Enthomogenisicrung ist durchaus ein sinnvolles Nebenprodukt. d. Globalhaushalte sollen die Regel werden, eine staatliche Finanzierung aufgrund planungssicherer volkswirtschaftlicher Daten wird angestrebt (Alternativentwurf S II).

Einer Vielzahl von neuen Bestimmungen im Alternativentwurf, die aufgrund der besonderen Programmatik der Partei Bündnis 90/Die Grünen verständlich sind Frauenförderung, Tierschutz, Forschungsorganisation, Mitbestimmung -, stehen viele Aspekte gegenüber, die parteiübergreifend Konsens finden, bloß aus taktischen Gründen nicht vom Regierungsentwurf und großkoalitionären Länder-SPDn eingebracht wurden (z.B. Tariföffnung, aber auch ein bundesrechtliches Verbot von Studiengebühren für alle Studiengänge, die zu Diplom, Magister, Bachelor und Master bzw. Promotion führen). Auch die Beendigung des unsinnigen Regelstudienzeitkonzepts $^{51}$, die Aufgabe der Staatsexamina außerhalb von Hochschulprüfungen, die Verbindung von Prüfungs- und Studienordnung etc. sind reformorientierte Details, die über eine bloß parteiprogrammatische Bindung hinausgehen.

Besonders wichrig ist die Festlegung des Alternativentwurfs auf cine Bindung an die Reform der Studienfinanzierung (Alternativentwurf $\$ 48$ Abs. s) und die Einführung cines Forschungsförderungsgesetzes in Bundesverantwortung gem. Art. $74 \mathrm{Nr}$. I3 GG (Alternativentwuif $₫ 48$ Abs. 4).

\section{Vorlänfiges Fazit}

1. Noch ist der Gesetzgebungsprozeß im vollen Gang, es stchen Verhandlungen und ggf. Auseinandersetzungen um folgende Bereiche mit Sicherheit an:

a. Zustimmungspflicht des Bundesrates (vom Bundesrat gefordert)

b. Bindung an BAFöG-Reform (teilweise von der SPD gefordert)

c. Verbot von Studiengebühren (vgl. die Bewertung der studentischen Proteste)

d. Tarifoffnung

Zu erwarten ist, daß der Regierungsentwurf durchgepeitscht wird und damit hinter allen Reformenvartungen zurückbleibt. Jede anderc Zusammensetzung einer Bundesregierung nach den Wahlen 1998 wird das HRG erneut zur Novellierung öffnen. Einige Länder warten dieses Datum nicht ab und novellieren bercits jetzt (Nordrhein-Westfalen, Niedersachsen, Hessen u. a.). Die 19. BAÏ̈̈G-Novelle wird nicht besser als die alte, so daß das Thema Studienfinanzierung auf der Tagesordnung bleibt. Die Bündnisgrünen bereiten einen Alternativentwurf-kompatiblen Gesetzcsentwurf mit einer Fondslösung vor. Die beiden großen Parteien haben mit ihtem inhaltsarmen Kompromiß eine Chance vcrtan und die Abkehr der studierenden Jugend von der verantwortlichen Politik und die immer schlechtere Rcputation dcurscher Universitäten im Ausland mitzuverantworten.

2. Als bescheidener Trost, auch im Hinblick auf die Kodifizierung von Hochschulrecht, bleibe der Befund, daß der Regierungsentwurf zu vicle Probleme offen und ungewollt dynamisch hält: Die Konflikte, zumal im Wahljahr, sind vorprogrammiert.

$31 \mathrm{Vgl}$. Konsens bet der Anhorung vom 8. 12. 1997 in Bundestag. 


\begin{tabular}{|c|}
\hline Hans Dahs (Hrsg.) \\
\hline Krininelle Kartelle? \\
\hline Zur Entstehungsgeschichte des neuen $§ 298$ StGB \\
\hline $\begin{array}{l}\text { Fachveranstaltung der Deutschen Strafverteidiger e.V. } \\
\text { am 14. Juni } 1996 \text { in Frankfurt am Main }\end{array}$ \\
\hline $\begin{array}{l}\text { Die Strafbarkeit von Submissionsabsprachen war seit Beginn der } \\
90 \text { er Jahre Gegenstand ausführlicher Diskussionen in Lehre und Pra- } \\
\text { xis. Auch durch den neu geschaffenen Straftatbestand des Ausschrei- } \\
\text { bungsbetruges ( } \$ 298 \mathrm{StGB} \text { ) sind die grundsätzlichen Probleme nicht } \\
\text { beseitigt. Die Fragen der Auslegung der neuen Bestimmungen lassen } \\
\text { sich nur sinnvoll beantworten, wenn man die Entstehungsgeschichte } \\
\text { und die Diskussion um die »Kriminellen Kartelle« kennt. Mit der } \\
\text { Kernfrage, ob es »Kriminelle Kartelle« gibt, befaßte sich der Deut- } \\
\text { sche Strafverteidiger e.V. auf einer Tagung. } \\
\text { Der Band enthält im ersten Teil die dort von Praktikern und Rechts- } \\
\text { lehrern gehaltenen Vorträge. Im zweiten Teil sind die - teilweise bis- } \\
\text { her unveröffentlichten - Entscheidungen des Landgerichts Frankfurt } \\
\text { am Main und des Bundesgerichtshofes zum sogenannten »Wasser- } \\
\text { bauverfahren« dokumentiert. Dem schließt sich eine ausführliche } \\
\text { Dokumentation des Gesetzgebungsverfahrens an, das im Sommer } \\
\text { 1997 zur Verabschiedung des neuen } § 298 \text { StGB führte. } \\
\text { Das Buch wendet sich an Richter, Staatsanwälte und Verteidiger } \\
\text { sowie an die Wissenschaft. }\end{array}$ \\
\hline $\begin{array}{l}\text { 1998, } 223 \text { S., brosch., } 69,-D M, 504,-\ddot{O} S, 62,50 \mathrm{sFr} \\
\text { ISBN 3-7890-5169-1 } \\
\text { (Schriftenreihe Deutsche Strafverteidiger e.V., Bd. 13) }\end{array}$ \\
\hline $\begin{array}{l}\text { NOMOS Verlagsgesellschaft } \\
76520 \text { Baden-Baden }\end{array}$ \\
\hline
\end{tabular}

